\title{
Exploring engagement with authors of randomised controlled \\ trials to develop recommendations to improve allocation
}

\section{concealment implementation and reporting [version 1; peer}

\section{review: 1 approved, 1 approved with reservations]}

\author{
Laura Clark (D), Natasha Mitchell, Catherine Hewitt, David Torgerson (iD)
}

York Trials Unit, Department of Health Sciences, University of York, UK, York, YO10 5DD, UK

V1 First published: 08 Feb 2021, 10:83

https://doi.org/10.12688/f1000research.27918.1

Latest published: 08 Feb 2021, 10:83

https://doi.org/10.12688/f1000research.27918.1

\section{Abstract}

Background: Reviews have consistently shown that allocation concealment is frequently implemented and reported suboptimally in randomised controlled trials (RCTs). This research aims to pilot engaging with authors of RCTs to explore their knowledge and understanding of allocation concealment implementation and reporting to ascertain areas and mechanisms for their improvement.

Methods: Authors that published RCTs in core clinical journals in one month in 2019 were identified. Authors were invited to complete questionnaires to elicit their views and experiences on the implementation and reporting quality focussing on allocation concealment. Methodological quality of allocation concealment was evaluated in this sample by assessing adherence to the Consolidated Standards of Reporting Trials (CONSORT).

Results: Reporting was suboptimal, with only $57 \%$ of allocation concealment methods reported to be implemented which were judged as adequate, with $18 \%$ using sealed envelopes and more than $40 \%$ not adequately reporting allocation methods. When exploring allocation concealment, implementation and reporting questionnaires were found to elicit a low response rate amongst authors of RCTs.

Discussion: Following analysis of the themes that emerged from the questionnaires, the main recommendations to improve reporting quality are: journals need to endorse, adhere and promote reporting guidelines, a methodologist could review methodological details of publications simultaneously to peer review, envelopes as a form of allocation concealment are poorly implemented and reported, so careful review of these is required, funders need to insist on more robust allocation concealment methods are employed if the RCT

\section{Open Peer Review \\ Approval Status ? \\ 2 \\ version 1 \\ 08 Feb 2021

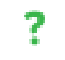 \\ view

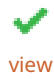 \\ 1. Fang Hua $(\mathrm{D}$, Wuhan University, Wuhan, \\ China \\ 2. Jelena Savovic iD, Bristol Medical School, Bristol, UK \\ Any reports and responses or comments on the article can be found at the end of the article.}


setting allows, and authors need to acknowledge their responsibility for transparent reporting of RCTs.

\section{Keywords}

reporting quality

This article is included in the Research on

Research, Policy \& Culture gateway.

Corresponding author: Laura Clark (laura.clark@york.ac.uk)

Author roles: Clark L: Conceptualization, Data Curation, Formal Analysis, Investigation, Methodology, Project Administration, Resources, Validation, Visualization, Writing - Original Draft Preparation; Mitchell N: Methodology, Supervision, Validation, Writing Review \& Editing; Hewitt C: Methodology, Supervision, Writing - Review \& Editing; Torgerson D: Methodology, Supervision, Writing Review \& Editing

Competing interests: No competing interests were disclosed.

Grant information: The author(s) declared that no grants were involved in supporting this work.

Copyright: @ 2021 Clark L et al. This is an open access article distributed under the terms of the Creative Commons Attribution License, which permits unrestricted use, distribution, and reproduction in any medium, provided the original work is properly cited.

How to cite this article: Clark L, Mitchell N, Hewitt $C$ and Torgerson D. Exploring engagement with authors of randomised controlled trials to develop recommendations to improve allocation concealment implementation and reporting [version 1; peer review: 1 approved, 1 approved with reservations] F1000Research 2021, 10:83 https://doi.org/10.12688/f1000research.27918.1

First published: 08 Feb 2021, 10:83 https://doi.org/10.12688/f1000research.27918.1 


\section{Introduction}

Allocation concealment is a key criterion of quality for a rigorously conducted randomised controlled trial (RCT). It is the most basic methodological piece of information that should be reported $^{1,2}$. Allocation concealment has been defined by the Consolidated Standards of Reporting Trials (CONSORT) group as:

'A technique used to prevent selection bias by concealing the allocation sequence from those assigning participants to intervention groups, until the moment of assignment. Allocation concealment prevents researchers from (unconsciously or otherwise) influencing which participants are assigned to a given intervention group.' Allocation Concealment, CONSORT.

A common misconception is that allocation concealment is the same as blinding ${ }^{3,4}$. Unlike allocation concealment, blinding is not always possible or even desirable to be carried out at all in RCTs ${ }^{5}$. This confusion may inadvertently lead to the use of poor allocation concealment.

Historically, sequentially numbered, opaque, sealed, envelopes (SNOSE) were used to conceal allocation sequences and are still widely used. Despite descriptions of 'relatively' tamper proof methods using envelopes ${ }^{6}$ they can still be easily subverted, such as by opening them in advance of patient recruitment, therefore findings from RCTs using envelopes ought to be open to greater levels of scrutiny ${ }^{3,7}$. If envelopes are to be used then methods minimising tampering need to be put into place and reported. A more secure approach for allocation concealment is to use central randomisation- this is a method which is more difficult to subvert as it separates the randomisation sequence generation from the allocation of participants ${ }^{3,7}$. Research has shown that trials with inadequate or unclear allocation concealment are associated with increased effect sizes of greater than $40 \%{ }^{1}$.

Unfortunately, RCT publications frequently do not report essential methodological factors in full, with many methodological elements, including allocation concealment, missing entirely $^{4,8}$ In 1996 the CONSORT statement was published, after a growing body of evidence identified a lack of consistent and high-quality reporting of $\mathrm{RCTs}^{9}$, with further revisions for different research designs ${ }^{8,10,11}$. Many, but not all, journals are now 'CONSORT endorsing' journals as they have adopted the CONSORT checklist for RCTs as a basic methodological checklist that authors should adhere to ${ }^{12}$.

Research recognises that insecure allocation concealment methods are still being implemented and reporting is still suboptimal despite CONSORT ${ }^{13-16}$. An area that needs exploring is understanding why personnel involved in the conduct of RCTs are implementing insecure methods and why these methods are still not being properly reported. Engaging with this group will enable targeted recommendations to be made to improve both the implementation and reporting of allocation concealment.

\section{Aims of this research}

- Pilot the use of a questionnaire to engage with authors of published RCTs to investigate their knowledge and understanding of allocation concealment implementation and reporting.

- Use these findings to ascertain areas and mechanisms which are acceptable to these authors to improve implementation and reporting of allocation concealment.

- Assess whether a questionnaire is an effective way to engage with authors of RCTs regarding methodological information to inform future research.

- Determine the reporting quality of allocation concealment in this sample by assessing the adherence to CONSORT.

\section{Methods}

We identified all RCTs published in one month in 2019 in the 'Abridged Index Medicus'. We do not have ethical approval to specify the month that the articles are published as this would identify the authors invited to participate in the research and not keep their identities secure. We undertook double data extraction of the randomisation method and allocation concealment method where the data was initially extracted and then second checked for any typological errors. We used this data to assess whether papers adhered to CONSORT. Table 1 details the criteria used.

\section{Table 1. Criteria used when judging randomisation and allocation concealment methods in included studies}

\begin{tabular}{|l|l|}
$\begin{array}{c}\text { Methodological } \\
\text { factor }\end{array}$ & \multicolumn{1}{c|}{ Methodological details determining the judgement } \\
\hline $\begin{array}{c}\text { Randomisation } \\
\text { method }\end{array}$ & This should be a truly random method to be adequate \\
\hline $\begin{array}{c}\text { Rllocation } \\
\text { concealment } \\
\text { allocation has occurred. }\end{array}$ & $\begin{array}{l}\text { If envelopes were used as an allocation concealment method they are only judged as adequate if they were } \\
\text { sequentially numbered sealed opaque envelopes (SNOSE), the personnel who created and opened them were both } \\
\text { stated, and these were not the same person. }\end{array}$ \\
\hline $\begin{array}{l}\text { Central randomisation was judged as adequate } \\
\text { Double-blind placebo-controlled trials were considered adequate as they are considered a robust trial design and } \\
\text { difficult to subvert. }\end{array}$ \\
\hline
\end{tabular}


We contacted corresponding authors via the email address cited in the publication and asked them to anonymously fill in a short questionnaire ${ }^{17}$ assessing their views on trial reporting. The questionnaire consisted of two questions with closed responses and one open ended question. The purpose of this questionnaire was to establish if authors could define, and identify what were adequate methods of, allocation concealment. Participants were asked to leave their email address if they consented to fill in an in-depth 18 item questionnaire. Both questionnaires were created and distributed using QUALTRICS ${ }^{18}$. A reminder email was sent two weeks after the initial, requesting participation in the survey.

The purpose of the second questionnaire was to elicit comprehensive knowledge and views on implementation, reporting quality and mechanisms to improve trial reporting quality in relation to allocation concealment.

Authors were sent the questionnaire and asked to respond within two weeks, after one week a reminder email was sent. The QUALTRICS survey was closed two months after the end date to ensure if there were late responses could still be submitted. For both questionnaires, where there were open/ free text boxes we performed a thematic analysis of the content of the questionnaire responses. The analysis was performed by LC and NM. Text was systematically examined, and themes identified by coding and categorising text according to key themes that emerged Data was initially organised in order to determine what participants knew about allocation concealment methodology, what method they had experience of using, if they knew what essential methodological information to report and what mechanisms could improve the reporting quality of RCTs and the opinions of suggestions to improve reporting quality of allocation concealment methodoloy These categories were further coded and mapped, as detailed participant responses were analysed to identify beliefs, experiences and barriers and facilitators to improving reporting quality, these lead to identifying and developing emergent themes.

An internal pilot was performed on each questionnaire with research staff in the York Trials Unit who had experience of being authors of RCTs and trial methodology, following piloting we reordered some of the questions but did not change the content

Ethical approval was granted by the University of York Health Sciences Research Governance Committee in March 2019. Information regarding study information and the process of consent was provided at the start of the questionnaire, submission of the completed questionnaire by the participant was considered as implicit consent. We provided contact details to enable recipients of the questionnaire to contact the research team with any queries, advised them on the length of time the survey was expected to take, that all responses were anonymous and findings from the survey would be published.

\section{Results}

In total, 95 RCTs were identified ${ }^{19}$, and $87 \%(n=83)$ were published in CONSORT endorsing journals. Of these, 94 had a corresponding author email address. The corresponding authors were a combination of clinicians and academics.

\section{Assessment of allocation concealment reporting}

Of the identified RCTs, $75.8 \%(n=72)$ reported the allocation sequence generation method and $71.6 \%(\mathrm{n}=68)$ reported the allocation concealment method (see Table 2 for details), with CONSORT endorsing journals reporting these methodological details more frequently.

Table 3 shows that almost half of RCTs in CONSORT endorsing journals did not report with sufficient clarity that a judgement could be made as to the robustness of allocation concealment. It also indicates that $21.1 \% \quad(n=20)$ were double blind placebo blind trials which were judged as adequate, of these only $60 \%$ $(n=12)$ reported their allocation sequence generation method.

\section{Allocation concealment methods: envelopes and central randomisation}

Envelopes were used to conceal the allocation sequence in $17.9 \%$ $(\mathrm{n}=17)$ of the included RCTs, with $52.9 \%(\mathrm{n}=9)$ describing the security of the envelope, $29.4 \% \quad(n=5)$ stating who prepared the envelopes, and $35.3 \%(n=6)$ stating who opened the envelope. The reporting quality of necessary methodological information was poor for studies using envelopes; only $17.6 \%$ $(n=3)$ were published adequately with all necessary information to assess the methodological rigour reported. Central randomisation was performed in $33(34.7 \%)$ RCTs.

Questionnaire analysis. In total, 12 (12.7\%) anonymous authors returned the shorter questionnaire. The participants

Table 2. Adherence to CONSORT when reporting randomisation and allocation concealment methods.

\begin{tabular}{|l|l|l|l|}
\hline & \multicolumn{2}{|c|}{$\begin{array}{c}\text { CONSORT } \\
\text { endorsing journal }\end{array}$} & \\
\hline Adherence to CONSORT & Yes n(\%) & No n(\%) & Total n (\%) \\
\hline $\begin{array}{l}\text { Allocation sequence } \\
\text { generation method } \\
\text { reported }\end{array}$ & $65(78.3)$ & $7(58.3)$ & $72(75.8)$ \\
\hline $\begin{array}{l}\text { Allocation concealment } \\
\text { method reported }\end{array}$ & $61(73.5)$ & $7(58.3)$ & $68(71.6)$ \\
\hline
\end{tabular}

Table 3. Assessments of allocation concealment adequacy in the included RCTs.

\begin{tabular}{|l|c|c|c|}
\hline $\begin{array}{c}\text { Allocation concealment } \\
\text { judgement }\end{array}$ & \multicolumn{2}{|c|}{$\begin{array}{c}\text { CONSORT } \\
\text { endorsing journal }\end{array}$} & \\
\cline { 1 - 4 } & Yes n(\%) & No n(\%) & Total n (\%) \\
\hline Adequate n(\%) & $49(59.1)$ & $5(41.6)$ & $54(56.8)$ \\
\hline Unclear n(\%) & $34(40.9)$ & $7(58.4)$ & $41(43.2)$ \\
\hline Total n(\%) & $83(87.4)$ & $12(12.6)$ & $95(100)$ \\
\hline
\end{tabular}


had a varied range of experience of how many RCTs they had conducted. Most participants $(n=8)$ had conducted $>3$ RCTs with one participant stating they had conducted $>300$ RCTs. Six authors $(6.4 \%)$ consented to being sent the second larger questionnaire and five authors completed this. From examining the email addresses, one participant was a clinician and five were academics. Figure 1 shows the summary of response rates to the questionnaires.

\section{Allocation concealment implementation}

All participants would use both central randomisation services and envelopes in future research. Of the participants, $80 \%$ $(n=4)$ believed that central randomisation should be the gold standard if appropriate to the trial design. Most participants supported the use of envelopes but did acknowledge that they were more at risk of subversion than central randomisation.

'I would say for the envelopes they are adequate if they are SNOSE. While they are adequate they are rather impractical sometimes, and they are not completely immune to subversion of course. Central randomisation is therefore preferred'(ID 2.5)

Participants were keen to emphasise that the choice of allocation concealment and randomisation is setting dependent.

'In my experience, it [selection of allocation concealment method] is dependent on the type of setting; e.g. in a busy clinical ward with $24 \mathrm{hr} / 7$ recruitment, envelopes are far more user-friendly, especially when relying on busy clinical staff to take consent and randomise. Alternatively, in a 9-5pm environment, telephone or web-based can be applied easily' (ID 2.2)

'Envelopes [are chosen] because it was the next best thing in lieu of central randomisation for sites with poor internet connection etc. Central randomisation [is chosen] because it's the best way' (ID 2.5)

\section{Envelope use}

There was a large amount of support for the implementation of envelopes as an allocation concealment method where appro-

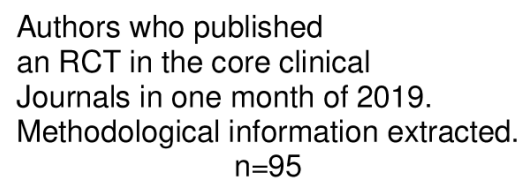

\begin{tabular}{|c|c|}
\hline & $\begin{array}{c}\text { Author without a } \\
\text { corresponding email address } \\
n=1\end{array}$ \\
\cline { 2 - 4 } & \\
\hline
\end{tabular}

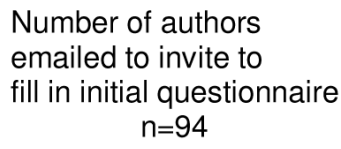

Figure 1. Summary of response rates to questionnaires. 
priate. The main reasons stated were trial setting, including the country and budget.

'[Reasons for envelope use] for cases where you are running trials in LMICs [low and medium income countries] with, e.g. limited internet access - envelopes may be more practical. I also used them in an older trial which did not have funding for a central randomisation service. I would only use them for these niche cases and not as routine.' (ID 2.5)

One participant selected that they did not think envelopes were an adequate allocation concealment method but they had used them in the past due to budget constraints.

\section{Central randomisation}

Central randomisation was accepted and described as being the 'best' with no elaboration as to why. One author considered the problematic reporting of central randomisation.

'Just saying its 'done centrally' [when reporting allocation concealment methods implemented] I also find problematic. If you can call someone up to randomise and they have an open allocation list, there is still a risk that there is some collusion between the two parties to ensure certain patients are randomised one way or the other' (ID 2.5)

\section{Essential methodological information knowledge}

We asked authors to list the top three methodological items to consider when conducting and reporting an RCT. Each response included the randomisation method employed with only one response stating that allocation concealment as important. Of the responses gained, $75 \%$ included blinding as an important methodological item to report, which could suggest that blinding and allocation concealment are being confused as past research has highlighted ${ }^{3,4}$.

From the short questionnaire $60 \%$ of participants stated they could not define allocation concealment. The most general way of defining allocation concealment was to discuss how the randomisation sequence should be hidden, but with no consideration as to why as defined by CONSORT only one participant included selection bias in their response.

'If done effectively it [allocation concealment] should reduce the risk of selection bias by preventing researchers from manipulating when individual participants are enrolled/randomised based on prior knowledge of the upcoming randomisation sequence' (ID

This suggests that many of those involved within the conduct of RCTs do not appear to see allocation concealment as an essential element to report.

There was a misconception that allocation concealment was the same as blinding the patients and outcome assessors by one participant, when asked to state what is important to report in RCTs.

\section{'Method of concealing the allocated treatments (blinding of patients and outcome assessors) [is important}

to report in RCTs]' (ID 2.4)

\section{Envelopes: methodology knowledge}

We determined whether participants were aware of how to adequately implement and report envelopes in RCTs by asking them to state what essential methodological information is necessary. No author knew all essential quality factors to include within a publication. Only one participant stated that the individual who opened the envelope should be reported, and no authors stated that it was essential to report who prepared the envelopes. All participants knew that envelopes should be sequentially numbered, and that this should also be reported. Participants were mostly aware of additional security measures regarding the envelope that could be taken and reported to decrease the risk of subversion.

'There are probably extra measures which may help to prevent subversion such as instructing staff to write the patient name etc. on the envelope prior to opening it, and ensuring that the seal is tamper proof - not e.g. a staple which can be carefully unpicked and re-stapled if someone was so motivated. A wax seal comes to mind although that sounds rather impractical too!' (ID 2.5)

Investigator integrity emerged as a theme here, with participants claiming that investigators had to implement envelopes correctly but not acknowledging what needed to be reported. This also supports the theme that there is a lot of focus on trial conduct rather than trial reporting, this is discussed further on in this publication

'Investigators have to use them [envelopes] properly. But investigators have to properly do all aspects of the trial. Basically, we always count on investigator integrity.' (ID 2.3)

'If I had to pick something [essential methodological information when reporting envelope use within an RCT] it would be processes to ensure adherence to correct use of the envelopes/prevent violation of the study protocol.' (ID 2.1)

\section{Improving reporting quality}

Participants recognised that reporting quality needed improving, and different mechanisms were explored:

Role of journal editor. Of those surveyed, $80 \%(\mathrm{n}=4)$ believed that the journal editor should ensure that key methodological information is reported within an RCT publication. It was also acknowledged that the editor does not necessarily have the expertise to do this.

'Journal editors should do their best to review methodological information but if they don't have sufficient methodology expertise across all experimental designs that may be included in submissions to their journal, then they are not the most appropriate person for this task' (ID: 2.1)

Word limits imposed by journals. We asked participants if they thought word limits imposed by journals impacted the amount 
of methodological information reported and thus the quality of the publication. There was agreement that although it could impact the quality, increasing the word count would most likely not help as the additional word limit may not be used to report the methodology.

'Increasing journal word counts is merely one mechanism to facilitate (but not ensure) more comprehensive reporting of methods/findings. At authors discretion they could simply use the additional word count to expand their discussion' (ID 2.1)

Pre-submission checks. We found there was minimal support from our surveyed population for journals to perform presubmission checks on a manuscript prior to full submission. We proposed that key methodological information of an RCT including allocation concealment and randomisation procedures were submitted to a journal for review. Only those manuscripts that include transparent and detailed methodological information would then be invited to submit their full manuscript. This could lead to improved reporting quality of allocation concealment and randomisation methodology.

Linked to this was the criticism of journals and the time it takes for the peer review process to be completed. This influenced participant opinions when considering improvements to reporting quality. Although all participants agreed there was a need to increase reporting quality, they did not want to add any additional barriers to slow down publications.

'It could add value but the length of the peer review process already substantially delays the release of scientific advances, and adding another rate-limiting step would exacerbate the delay'

$$
\text { (Paper ID 2.1) }
$$

'All these points are important [to improve reporting quality] but I don't want more "process" [when trying to submit a manuscript to a journal]' (ID 2.3)

Methodologist. The largest support was given to a methodologist always assessing the methods section of any publication submitted to a journal, similar to the statistical review some journals subject manuscripts to. Support was given to this if it was performed in a streamlined way and done simultaneously whilst the manuscript was going through the peer review process; participants were not supportive of any method of improve=ing trial reporting that they perceived to hold the process up further.

'There is no reason why specialised methodology review could not be included as part of the standard external peer review process.'

Role of Funders. Support for funders allocating specific funding for a third-party randomisation service if the RCT setting and design suited this emerged.

'These [allocated funding for a third-party randomisation service] can help ensure robust methods before trial conduct, and thus the quality of the trial and report' (ID 2.2)

There was also a suggestion for funding organisations to mandatorily require and fund external independent assessors
'It would strengthen the robustness of any trial results if funding organizations would demand and financially support external independent assessors' (ID: 2.4)

Both of these points however fail to recognise that robust trial design and methodology does not necessarily mean the RCT publication will be written to reflect this; these suggestions will not prevent poor reporting quality they only impact trial conduct. This theme is considered later in this manuscript.

\section{Awareness of and adherence to guidelines}

All participants stated that they used CONSORT guidelines when writing their RCT. Participants emphasised that some authors may not be aware of CONSORT, and that even though some journals do endorse the CONSORT statement there is a difference in how they are adhered to between journals which may impact reporting quality.

\section{'I think that many clinicians may be unaware of the full consort statement' (ID 2.4)}

'Journals should adhere strictly to appropriate research reporting guidelines (e.g. CONSORT for RCTS)'. (ID 2.1)

There was universal support for CONSORT guidelines and strong agreement that there were adequate guidelines and support to aid the robust and transparent publication of research; rather it was adherence to the existing guidelines that is needed, with a focus on researchers ensuring this occurred.

'Guidelines for acceptable processes are readily available and reporting of that information is commonly required in key research reporting guidelines. If all researchers adhere to those recommendations we should have very little risk of selection bias in published research..... Spend the effort/time/money advocating for

better adherence to existing reporting guidelines.' (ID 2.1)

Linked to this was that there were already mechanisms in place to enable trials to be reported adequately and that these need to be adhered to.

'Many journals require submission of a supplementary document demonstrating adherence to an appropriate reporting guideline, which commonly directs readers to the appropriate section/page of a manuscript to find relevant information.' (ID 2.1)

\section{The translation of trial design and conduct to the} publication

A recurring theme was the idea that if the trial was conducted robustly it would lead to a robust publication automatically. A lot of emphasis was placed on those researchers conducting themselves correctly, with integrity and following the protocol with the implication this would lead to a robust publication.

'These options [suggestions to aid reporting quality] can help ensure robust methods before trial conduct, and thus the quality of the trial and report' (ID 2.2)

'[to improve reporting quality] ensure adherence to correct use of the envelopes/prevent violation of the study protocol' (ID: 2.1) 


\section{Discussion}

Our research highlighted again that there is suboptimal implementation and reporting of allocation concealment. Only 57\% of RCTs published in CONSORT endorsing journals contained adequate allocation concealment methods.

Analysis of the questionnaire data indicated that some authors could not accurately define allocation concealment, and it was at times confused with blinding. Only one participant considered it to be within the top three methodological elements of an RCT to report, indicating that it may not be reported adequately as it is not seen as a priority.

To improve the reporting of allocation concealment there was support for a methodologist to review submitted articles to ensure essential methodological information was reported. There are a number of barriers to operationalise this particular mechanism. One solution could be to nominate a trial team member to be the guarantor for required methodological information on submission of a manuscript, if journals do not have the capacity to support this specialist process.

The views of this sample of participants suggest that envelopes are going to be employed as a method of allocation concealment in future research for a variety of reasons such as budget, but authors had poor knowledge on which methodological factors should be reported.

Central randomisation was accepted as the gold standard but cost was a barrier for its implementation. Authors did not consider that choosing a less expensive allocation concealment method could result in inadequate methods being employed, which, could lead to research that is judged as a high risk of bias $^{20,21}$. We agree with the discussion point raised by a participant stating that randomisation that was 'performed centrally' does not give the reader enough information on what was carried out, this is suboptimal reporting.

\section{RCT author engagement}

This research demonstrated that a survey elicits a low response rate when engaging with authors regarding allocation concealment knowledge, and different mechanisms to engage are needed. The corresponding author of a publication in healthcare is frequently a clinician and not a methodologist, which could explain the low response rate to a questionnaire about methodological information. We intentionally did not limit word counts within the free text boxes to enable full exploration and elicitation of views, this enabled us to capture rich data. Despite the response rate the data was consistent amongst the participants, those that did respond showed high engagement with the topic and this has enabled a targeted list of recommendations to be made on improving the implementation and reporting of allocation concealment for this sample.

\section{Limitations}

There are many methodological quality factors that could have been addressed when assessing reporting quality. Nevertheless, allocation concealment was our focus due to it being the central part of an RCT. There was a low response rate to the questionnaire with the majority of responses from academics rather than clinicians, which limits the generalisability of the findings amongst the RCT author population. The low survey response rate is a finding that has been observed in other research with a trend of decreasing participant engagement over time $^{22-24}$, it is postulated that frequent survey requests and work schedules may influence participation, particularly with health professionals ${ }^{25}$. We did not repeat the survey to increase the sample size as an aim of this research was to assess the effectiveness of this method on author engagement, the response rate demonstrated that this method did not yield a high participation rate for this research area.

\section{Future research}

Further work into how best to engage with RCT authors is necessary, interviews may be a better method to engage and unpick what is underpinning suboptimal implementation and reporting of allocation concealment. Identifying who is the methodologist within the author list and engaging with this individual may enable us to determine how the methods section was written and reported and why poor reporting was occurring by examining the internal editing processes that occurs within research teams, including any comments received from reviewers and methodological information that could have been lost during the publication process. Clinicians were under represented in this research, and exploring their views may elicit different mechanisms to improve allocation concealment implementation and reporting.

Work into exploring different mechanisms and reporting platforms where there is an instant publication and a transparent reviewing process is recommended (such as with F1000 Research) which could lead to recommendations in speeding up reviewing time and processes which was highlighted as an issue with this surveyed sample.

\section{Recommendations and conclusion}

This research highlighted that there needs to be greater knowledge of allocation concealment amongst authors of RCTs, as well as the understanding that securely implementing it does not automatically generate a well written RCT publication.

From the findings of this research to improve allocation concealment implementation and reporting we recommend:

- CONSORT is endorsed and adhered to by each journal as it was accepted by all participants in this research

- Trials using envelopes need to be discouraged and if they are to be used then they should be robustly implemented and reported with careful checking that key methodological information is included in publications, as knowledge around this was suboptimal

- Funders assess proposed methodology and insist on the use of robust allocation concealment methods if the RCT setting allows (such as central randomisation) 
- If central randomisation is implemented full methodological details are reported within the publication, including the names of external centres involved and the method of randomisation (simple, restricted) and details such as stratification variables and block size.

- $\quad$ Authors need to take responsibility for ensuring key methodological factors are included in their RCT publications and should report all methods transparently even if not methodologically robust

- A methodologist reviews the RCT publication to ensure key methodological information is included simultaneously to the peer review process. Although this is a recommendation based from the findings of this research the difficulties in the practicalities is discussed.

\section{Data availability}

Underlying data

Figshare: Methodological details of included studies

https://doi.org/10.6084/m9.figshare.13550348.v1 $1^{19}$

This project contains the following underlying data:

- Methodological details of included studies.csv (Methodological details reported in publications of randomised controlled trials included in the study).

Data are available under the terms of the Creative Commons Attribution 4.0 International license (CC BY 4.0).
We can only currently share the questionnaires sent to participants and the non-identifiable data from the data extraction of the allocation concealment judgement where we identified included studies. We are only able to share the data extracted related to the journal CONSORT endorsement, quality judgements we made on the allocation concealment methods and the breakdown of different methods used (such as central randomisation and envelopes use), as to share the full extracted methods section and title from each included paper would enable the paper and therefore participants to be identified.

If readers or reviewers would like to apply for access to the data they would have to contact Laura Clark at laura.clark@ york.ac.uk. We are required to liaise with the University of York Health Sciences Research Governance Committee (https:// www.york.ac.uk/healthsciences/research-information/rsg/) to request permission to share the requested data and adhere to the conditions under which they will grant access.

Extended data

Figshare: Questionnaire to authors

https://doi.org/10.6084/m9.figshare.13550351.v1 $1^{17}$

This project contains the following extended data:

- Questionnaires used in study.docx (Questionnaire sent to authors)

Data are available under the terms of the Creative Commons Attribution 4.0 International license (CC BY 4.0).
1. Jüni $P$, Altman $D G$, Egger M: Assessing the quality of controlled clinical trials. BMJ. 2001; 323: 42-46.

Publisher Full Text

2. Schulz KF: Subverting randomization in controlled trials. JAMA. 1995; 274(18): 1456-8.

PubMed Abstract | Publisher Full Text

3. Schulz KF, Chalmers I, Hayes RJ, et al.: Empirical evidence of bias. Dimensions of methodological quality associated with estimates of treatment effects in controlled trials. JAMA. 1995; 273(5): 408-412.

PubMed Abstract | Publisher Full Text

4. Schulz KF, Grimes DA: Blinding in randomised trials: hiding who got what. Lancet. 2002; 359(9307): 696-700.

PubMed Abstract | Publisher Full Text

5. Forder PM, Gebski VJ, Keech AC: Allocation concealment and blinding: when ignorance is bliss. Med J Aust. 2005; 182(2): 87-9.

PubMed Abstract | Publisher Full Text

6. Doig GS, Simpson F: Randomization and allocation concealment: a practical guide for researchers. J Crit Care. 2005; 20(2): 187-191.

PubMed Abstract | Publisher Full Text

7. Higgins JPT, Altman DG, Sterne JAC: Chapter 8: Assessing risk of bias in included studies. In: JPT H and Green S, (eds.). The Cochrane Collaboration, 2011 Cochrane Handbook for Systematic Reviews of Interventions Version 510 (updated March 2011), 2011.

Reference Source

8. Schulz KF, Altman DG, Moher D, et al.: CONSORT 2010 statement: updated guidelines for reporting parallel group randomised trials. BMC Med. 2010; 8: 18.

PubMed Abstract | Publisher Full Text | Free Full Text

9. Begg C, Cho M, Eastwood S, et al.: Improving the quality of reporting of randomized controlled trials. The CONSORT statement. JAMA. 1996; 276(8):
637-9.

PubMed Abstract | Publisher Full Text

10. Moher D, Schulz KF, Altman DG: The CONSORT statement: revised recommendations for improving the quality of reports of parallel-group randomised trials. Lancet. Elsevier. 2001; 357(9263): 1191-4. PubMed Abstract | Publisher Full Text

11. Moher D, Schulz KF, Altman D, et al:: The CONSORT statement: revised recommendations for improving the quality of reports of parallel-group randomized trials. JAMA. 2001; 285(15): 1987-1991.

PubMed Abstract | Publisher Full Text

12. Altman DG, Simera I, Hoey J, et al.: EQUATOR: reporting guidelines for health research. Open Med. 2008; 2(2): e49-50. PubMed Abstract | Free Full Text

13. Mitchell A, Moe-Byrne T, Cunningham-Burley R, et al:: Poor allocation concealment methods are associated with heterogeneity in age and statistical significance of the primary outcome: Review of recent trials published in four general medical journals. J Eval Clin Pract. 2020; 26(4): 1316-1319.

PubMed Abstract | Publisher Full Text

14. Hewitt C, Hahn S, Torgerson DJ, et al:: Adequacy and reporting of allocation concealment: review of recent trials published in four general medical journals. BMJ. 2005; 330(7499): 1057-1058. PubMed Abstract | Publisher Full Text | Free Full Text

15. Turner L, Shamseer L, Altman DG, et al.: Does use of the CONSORT Statement impact the completeness of reporting of randomised controlled trials published in medical journals? A Cochrane reviewa. Syst Rev. 2012; 1: 60. PubMed Abstract | Publisher Full Text | Free Full Text

16. Hopewell S, Dutton S, Yu LM, et al.: The quality of reports of randomised trials in 2000 and 2006: comparative study of articles indexed in PubMed. 
BMJ. 2010; 340: c723

PubMed Abstract | Publisher Full Text | Free Full Text

17. Clark L, Mitchell N, Hewitt C, et al.: Questionnaire to authors. figshare. Figure. 2021

http://www.doi.org/10.6084/m9.figshare.13550351.v1

18. QUALTRICS: Qualtrics. 2019.

19. Clark L, Mitchell N, Hewitt C, et al.: Methodological details of included studies.csv. figshare. Dataset. 2021.

http://www.doi.org/10.6084/m9.figshare.13550348.v1

20. Higgins JPT, Altman DG, Sterne JAC: Chapter 8: Assessing risk of bias in included studies. In: JPT H and Green S, (eds.). The Cochrane Collaboration, 2011 Cochrane Handbook for Systematic Reviews of Interventions Version 510 (updated March 2011). 2011.

Reference Source

21. Higgins JPT, Savović J, Page MJ, et al.: Assessing risk of bias in a randomized trial. Cochrane Handbook for Systematic Reviews of Interventions. 2019; 205-228. Publisher Full Text
22. Blanco D, Hren D, Kirkham J, et al.: A survey exploring biomedical editors' perceptions of editorial interventions to improve adherence to reporting guidelines. [version 3; peer review: 3 approved]. F1000Res. 2019; 8: 1682. PubMed Abstract | Publisher Full Text | Free Full Text

23. Mulligan A, Hall L, Raphael E: Peer review in a changing world: An international study measuring the attitudes of researchers. J Am Soc Inf Sci Technol. 2013; 64(1): 132-161. Publisher Full Text

24. Price A, Schroter S, Clarke M, et al.: Role of supplementary material in biomedical journal articles: surveys of authors, reviewers and readers. $B M$ J Open. 2018; 8(9): e021753.

Open. 2018; 8(9): e021753.
PubMed Abstract | Publisher Full Text | Free Full Text

25. Flanigan TS, McFarlane $\mathrm{E}$, Cook S: Conducting survey research among physicians and other medical professionals - a review of current literature. In: Proceedings of the Survey Research Methods Section, American Statistical Association. 2008; 4136-4147.

Reference Source 


\section{Open Peer Review}

\section{Current Peer Review Status:}

\section{Version 1}

Reviewer Report 27 October 2021

https://doi.org/10.5256/f1000research.30872.r96107

(C) 2021 Savovic J. This is an open access peer review report distributed under the terms of the Creative Commons Attribution License, which permits unrestricted use, distribution, and reproduction in any medium, provided the original work is properly cited.

\section{Jelena Savovic}

Population Health Sciences, Bristol Medical School, Bristol, UK

Informative study, but based on a very small sample of authors and very poor response rate. Methods used are appropriate.

I answered 'Partly' to the Q, 'Are all the source data underlying the results available to ensure full reproducibility?' because the month of the search for RCTs could not be revealed due to ethical reasons. This would make the study more difficult to reproduce. However, not disclosing the participants is more important than being able to reproduce the exact study the authors did. Interested parties would be able to reproduce the study for a different publication period, which would be a more useful endeavour.

Although the recommendations made are sensible, is it really possible to make recommendations based on responses from 6 authors? Perhaps a stronger caveat to these recommendations would help? This was the reason I answered 'Partly to the last reviewer question. I feel that the main finding is that engaging RCT authors via surveys does not work.

In the text when the $\%$ of participants are given, can the actual numbers be given as well please? E.g. 'From the short Q-re 60\% of participants stated...' to 'From the short Q-re 7 (60\%) of participants stated...' or to state 7 (out of 12). I find this especially important with such small numbers.

The findings of the first part of the study, based on the analysis of reporting in 95 RCTs published in one month in 2019, are perhaps more interesting. There are some additional items I would be interested to read about, that the authors may be able to extract from their sample of 95 RCTs:

Comparative adequacy of reporting by type of corresponding author (clinician vs academic). Perhaps this could identify some training needs in these groups.

Under Allocation concealment methods: envelopes - Can you extract data on how many of the RCTs that used the envelope method described any sort of process for ensuring that the next envelope is not opened until the next participant is irreversibly enrolled? I find that this 
part of the concealment process for envelopes is often ignored and almost never reported. It would be interesting to see if any of the RCTs in your sample reported it.

Minor issues:

There are several spelling errors/typos and missing punctuation in the manuscript. - e.g:

In Methods, para 4:

"...could improve the reporting quality of RCTs and the opinions of suggestions to improve reporting quality of allocation concealment methodoloy These categories were further coded and mapped, as detailed..." - "methodoloy" is misspelt and there is a missing period after it.

In the Methods para 5:

"An internal pilot was performed on each questionnaire with research staff in the York Trials Unit who had experience of being authors of RCTs and trial methodology, following piloting we reordered some of the questions but did not change the content " - The sentence should break after 'methodology' and the new sentence to start with 'Following'. Also the period is missing at the end of the para.

Is the work clearly and accurately presented and does it cite the current literature? Yes

Is the study design appropriate and is the work technically sound?

Yes

Are sufficient details of methods and analysis provided to allow replication by others? Yes

If applicable, is the statistical analysis and its interpretation appropriate? Yes

Are all the source data underlying the results available to ensure full reproducibility? Partly

Are the conclusions drawn adequately supported by the results? Partly

Competing Interests: No competing interests were disclosed.

Reviewer Expertise: Evidence synthesis, RCTs, non-randomised studies, bias, epidemiology, metaepidemiology.

I confirm that I have read this submission and believe that I have an appropriate level of expertise to confirm that it is of an acceptable scientific standard.

Reviewer Report 17 May 2021

https://doi.org/10.5256/f1000research.30872.r84202 
(C) 2021 Hua F. This is an open access peer review report distributed under the terms of the Creative Commons Attribution License, which permits unrestricted use, distribution, and reproduction in any medium, provided the original work is properly cited.

\section{Fang Hua}

Centre for Evidence-Based Stomatology, School \& Hospital of Stomatology, Wuhan University, Wuhan, China

This is an interesting and important study regarding allocation concealment. The authors assessed the reporting and methodological quality of allocation concealment among RCTs published in "core clinical journals" in one month of 2019. They also carried out online questionnaires to investigate the knowledge of the RCT authors about allocation concealment. The study was generally well-conducted, but the article could be improved in the following aspects.

1. A main limitation of this study is its sample size, with only 95 RCTs and 5 participants included. These need to be properly stated in the limitation paragraph. The authors explained that they did not repeat the survey to increase sample size as it was an aim to assess the effectiveness of questionnaire survey on author engagement. If this is the case I suggest abridging the part of introducing findings of this survey, as the findings were based on a very small sample and might not be very representative / reliable anyway. In addition, please provide the rationale for sample size determination, i.e. why did you decide to include RCTs published in one month of 2019, and was the sample size calculated a priority.

2. The authors mentioned that they used CONSORT to assess methodological quality, but CONSORT is a reporting guideline. Maybe it should be stated that methodological quality was assessed using a checklist developed based on CONSORT.

3. The authors used the term "implementation and reporting of allocation concealment" for several times. "Conduct" or "execution" might be a better word than "implementation", as the latter has a different meaning in "implementation science".

4. Abstract - the total number of included RCTs should be included in the Results section; the sentence in Discussion section is quite long and difficult to follow.

5. Keywords - please add more keywords to facilitate indexing and identification of this paper.

6. Methods - "We identified all RCTs published in ..."-how were these RCTs identified (e.g. electronic search or hand search the journals' issues), and when was the last search conducted? If an electronic search was performed, the search strategy needs to be provided.

7. Table 1 - “Double-blind placebo-controlled trials were considered adequate as they are considered a robust trial design and difficult to subvert". This criterion seems inappropriate, as according to Cochrane Handbook allocation concealment is to avoid selection bias, while blinding is related to performance \& detection biases. Please provide rationale for this criterion, or add this to the limitations paragraph.

8. Results - It will be better if the characteristics of included RCTs were described, so that 
readers can better understand the context of these findings.

9. References \#7 and \#20 are the same.

Is the work clearly and accurately presented and does it cite the current literature?

Yes

Is the study design appropriate and is the work technically sound?

Yes

Are sufficient details of methods and analysis provided to allow replication by others?

Yes

If applicable, is the statistical analysis and its interpretation appropriate?

Yes

Are all the source data underlying the results available to ensure full reproducibility? Yes

Are the conclusions drawn adequately supported by the results?

Partly

Competing Interests: No competing interests were disclosed.

Reviewer Expertise: Clinical epidemiology, evidence-based medicine

I confirm that I have read this submission and believe that I have an appropriate level of expertise to confirm that it is of an acceptable scientific standard, however I have significant reservations, as outlined above. 
The benefits of publishing with F1000Research:

- Your article is published within days, with no editorial bias

- You can publish traditional articles, null/negative results, case reports, data notes and more

- The peer review process is transparent and collaborative

- Your article is indexed in PubMed after passing peer review

- Dedicated customer support at every stage

For pre-submission enquiries, contact research@f1000.com 